\title{
Asynchronous Dual Radio Opportunistic Beacon Network Protocol for Wildlife Monitoring System
}

\author{
Eyuel D. Ayele, Nirvana Meratnia, Paul J.M. Havinga \\ Pervasive Systems Research Group \\ University of Twente, Enschede, The Netherlands \\ Email: \{e.d.ayele,n.meratnia,p.j.m.havinga\}@utwente.nl
}

\begin{abstract}
Currently there are several technologies such as opportunistic wireless sensor networks deployed to monitor wildlife. Due to network complexity and lack of full connectivity, the collected data is often either saved on internal memory for later off-line data transfer or relayed to sink nodes. In this paper, however, we introduce an asynchronous dual interface opportunistic beacon network for animal monitoring. Unlike conventional opportunistic networks which are based on multi-copy data replication techniques, our approach utilizes an optimized single-copy beacon data transmission to achieve high energy efficiency. Furthermore, the collected data is aggregated and relayed to the central system by leveraging a low power and long range radio to provide high connectivity coverage. This approach will allow to facilitate ultra-low power IoT devices to be deployed for sustainable wildlife monitoring applications. We evaluate the proposed approach in an actual animal movement use-case scenario. The results indicate that the proposed approach outperforms the traditional opportunistic networks in-terms of energy consumption and packet delivery ratio.
\end{abstract}

Keywords-Wildlife monitoring, LoRa, BLE, Beacon

\section{INTRODUCTION}

During the last few years, Internet of Things (IoT) has received increasing worldwide attention for its numerous applications, consequently, number of IoT devices deployed are growing steadily. One of the practical applications of IoT is wildlife monitoring, in which the activities of wild animals are monitored by employing heterogeneous sensors (e.g. gyroscope, accelerometer, etc) either on collars or buried underground $[1,2]$. The movement behavior of wild animals often show a sparse and con-species (clustered) movement behavior. This behavior results in frequent change in the network topology, which gives rise to challenges in peer-to-peer network connectivity and energy management [3]. Wildlife monitoring systems (WMS) need to monitor the animal herd physiological activities in real-time as well as to provide network services such as localization, proximity detection, data pre-processing, and cluster nodes management. This requires (i) high energy efficiency, since the sensors used in a WMS will operate with a limited source of energy, (ii) good reliability to avoid false alarms, and (iii) low latency for a responsive WMS.

Based on movement behavior of wild animals and target application requirements, numerous opportunistic networks could be potential candidates for WMS. Conventional mobility adaptive opportunistic networks e.g Epidemic or PRoPHET [4, 5] are not suitable for WMS applications, mainly due to their multi-copy replication approach that leads to a high resource consumption in-terms of such as energy and data storage space. Moreover, when wild animals depict sparse and conspecific movements, this results in a sporadic and unstable end-to-end connectivity among nodes, hence, reducing network wide data reliability. Even-though mobility is considered as the fundamental facilitator for information dissemination in opportunistic networks, recent works have revealed that current opportunistic protocols perform wors than expected for sparsely mobile networks with non-deterministic movement $[4,5]$. For instance, Epidemic [4], and PRoPHET [6], offer a high data delivery ratio at the expense of high network overhead and latency [5]. Spray and Wait (SnW) [4] on the other hand results in low latency but has high network overhead [5].

Several existing opportunistic network based wildlife monitoring projects exists, for instance, ZebraNet [7], Rat Watch [8]. These projects, implement opportunistic networks by leveraging an Epidemic flooding, which is prone to low delivery ratio and high latency [4]. Moreover, they lack the low power data aggregation backbone network to relay data to a central system. Instead, they often utilize offline data gathering or cellular and satellite based systems [9]. However, the inherently high energy cost and intolerable communication latency make these approaches less attractive.

In this paper we present an asynchronous dual radio opportunistic beacon network for wild-life monitoring system (WMS). Our proposed opportunistic beacon network leverages a high data rate BLE radio in a low power long range LoRaWAN network [3]. Since utilizing LoRaWAN for long range data relaying, introduces a $1 \%$ duty-cycle communication restriction, which impacts the real-time latency requirement. We, therefore, address this challenge by utilizing dual interface, to provide a wider control over the trade-offs in energy versus latency, and by not sending all raw data to the LoRaWAN server. Instead, data pre-processing within the animal herds or groups is applied before relaying the data to the central server. This is mainly because data processing is computationally cheaper than data transmission. This ultimately, reduces the implementation complexity of the solution [3]. As a result the proposed solution will be energy efficient, scalable and responsive compared to traditional opportunistic protocols. In this research work, we make the following contributions:

- We propose a simple asynchronous dual interface opportunistic network protocol for animal monitoring.

- We evaluate and compare the protocol with existing asynchronous opportunistic protocols. 
The rest of the paper is organized as follows: Section II describes existing opportunistic network solutions. Section III presents the proposed network protocol and design approaches for the wildlife monitoring system. Section V discuses the use case scenario and presents the large scale evaluation results. Finally, a concluding remark and future research challenges are described in Section VI.

\section{RELATED WORK}

In conventional opportunistic networks (e.g Epidemic or ProPHET [4]), deciding where data should be sent to is often straight forward; i.e. the data is forwarded to the next neighbor along the path to the sink usually through the shortest path. Moreover, the gathered data is transferred to a sink using either fixed or mobile intermediate nodes used as relays. Traditional opportunistic protocols will perform poorly in WMS scenarios where the network is sporadically connected due to animal mobility pattern [7]. Currently, several research works have improved data collection based on opportunistic sensing with mobile network infrastructure support [6]. However, unless standard mobility model is considered, they often result in low delivery ratio and high latency [7, 8]. Thus, an efficient data dissemination protocol is needed for a newly surfacing wildlife monitoring applicaions with mobile sensor nodes.

A common similarity among opportunistic protocols is that, they are based on store-carry-forward (SCF) data replication technique, however, they differ in their approach to optimize and restrict the degree of replication. For instance, Direct Delivery (DD) algorithm enables a node to directly exchange a data to the destination in range [5]. After the communication is finished the sender node erases the replicated message to avoid local buffer queue overflow. Hence, the direct delivery scheme is based on single replication routine which often results in low delivery ratio.

Epidemic protocols spread data through out the network similar to microorganism infection [4]. When end-devices are in contact, they exclusively replicate multiple copies of data to the near by receiver nodes. These processes will be repeated through the network when nodes are in their range of communication, and ultimately data will reach the intended sink node. These epidemic approaches result in a high data reliability, however, they will drastically deplete sensor node resources, e.g. energy and data storage space [5].

To solve the problem in Epidemic protocol, Balasubramanian et al. [10] treated routing as a resource allocation problem and proposed RAPID protocol. The authors used an in-band control channel to exchange various metadata including expected contact time with other nodes, list of data delivered, and average size of past transfer events. RAPID essentially defines a per-data utility function, in which the data is replicated in such a way that it locally optimizes the marginal utility.

Similarly, ProPHET [6] is introduced to estimate data delivery probability for every contact with a destined node before sending a data. Moreover, Spyropoulos et. al $[6,11]$ introduced the Spray and Wait (SnW) protocol, by limiting and optimizing the number of data replication for every data forwarding. ProPHET and $\mathrm{SnW}$ have been found to reduce the energy consumption and increase the data delivery ratio considerable compared to Epidemic like protocols.

\section{PROTOCOL DESIGN}

In this section, we introduce an asynchronous opportunistic beacon network protocol for wildlife monitoring system. In order to carry out fine-grained monitoring applications, the proposed beacon protocol introduces a versatile and lightweight connectivity scheme, called opportunistic beacons, that expedites rapid and energy-efficient information sharing between mobile sensor devices without requiring connection establishment and complex configurations.

\section{A. Beacon Communication Scheme}

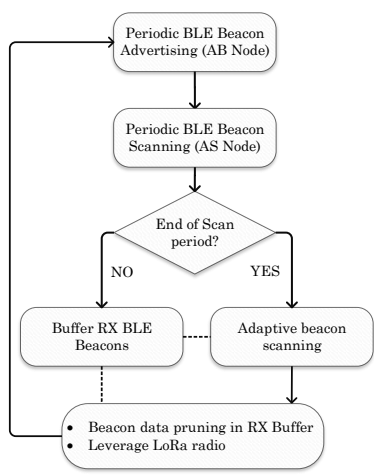

Fig. 1: AB-to-AS beacon communication scheme.

In the proposed protocol, there are three network device types: (i) AS- Animal Scanner, (ii) AB- Animal Broadcaster, and (iii) LG- LoRaWAN Gateway. $\mathrm{AB}$ is a BLE beacon broadcasting node, while AS is a BLE scanner node, which listens for BLE beacons in the surrounding. LG is a LoRaWAN gateway node which receives a LoRa packets from AS node. The overview of the proposed beacon protocol scheme is shown in Figure 1. The AB-AS beacon communication includes three main schemes: (i) periodic beacon advertising by $\mathrm{AB}$ nodes, (ii) periodic beacon scanning by $\mathrm{AS}$ nodes, and (iii) beacon data pruning and aggregation by AS node. In what follows we elaborate on each of these three schemes.

1) Periodic beacon advertising: Beacon discovery is initiated by $A B$ devices periodically sending beacon data and $A S$ devices scanning for nearby beacons by listening for data in advertising channel. AB node uses periodic asynchronous BLE mode to broadcast data to AS scanner within range. ABAS BLE beacon communication is a many-to-many ( $m$-to-m) transfer [12]. This sequence of events is called an advertising event. Advertising activities could occur at regular intervals called broadcast interval.

2) Periodic beacon scanning: As AS node commences its scanning operation, it listens and buffers the number of beacons it has received during its current and previous scanning window. Scanning activities occur at regular intervals. At the end of every scan window, AS node adapts the duration of the scanning interval according to the number of beacons received in the current and previous scanning. AS node changes to LoRa 
interface to relay beacons to LG node. This enables the AS node to considerably decrease the energy consumption.

3) Beacon data pruning and leverage LoRa radio: AS nodes have a dual radio, i.e. they utilize BLE as a short-range asynchronous interface and LoRa as an LPWAN solution, while $\mathrm{AB}$ nodes only have a BLE bearer. The AS enddevices use short range $\mathrm{BLE}$ to receive beacons from $\mathrm{AB}$, and long range LoRa radio to send aggregated data to LoRaWAN Gateway (LG). While obeying the $1 \%$ duty-cycle of limitation of LoRa radio. Readers are referred to [12-14] for more details on LoRa and BLE technology. After finishing sending LoRa packets, AS node switches back to listening the incoming BLE beacon data from $\mathrm{AB}$ nodes.

\section{B. Operation of $A B$ and $A S$ Node}

\section{LoRaWAN Network}

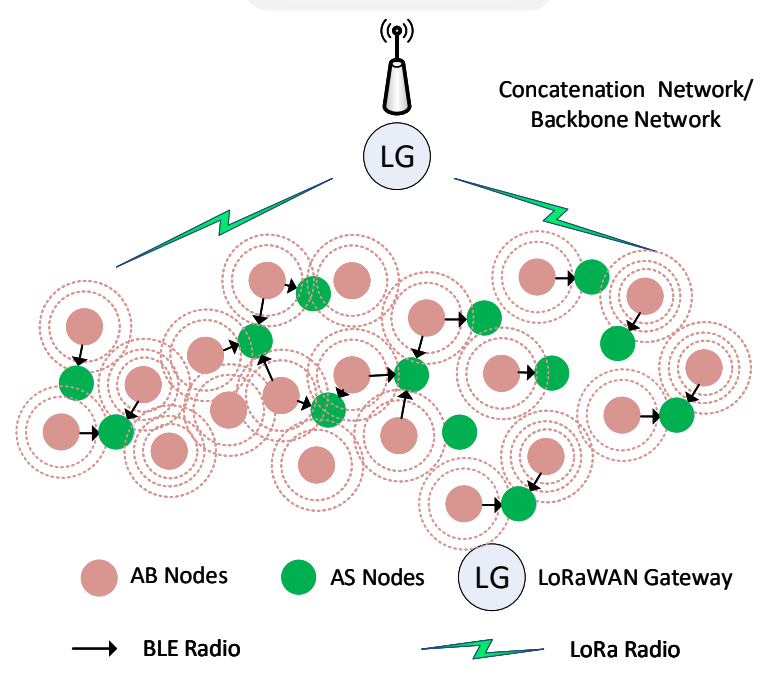

Fig. 2: A dual radio beacon network. Short range radio (BLE) is utilized for opportunistic beacon network mode $(A S-A B)$ realization and LoRa radio is used to link to LoRaWAN Gateway (LG). Device types are: (i) AS-Animal Scanner, (ii) AB-Animal Broadcaster, and (iii) LG-LoRa Gateway.

The proposed opportunistic beacon network is shown in hierarchical layout in Figure 2. The bottom tier consists of a network of AS-AB devices. The detailed operation of $\mathrm{AB}$ and AS nodes are show in Figure $3 a$ and Figure $3 b$ respectively.

As shown in Figure $3 \mathrm{a}, \mathrm{AB}$ node starts beacon advertising with a single copy beacon data by periodically (with $T_{B C}^{+}$) interrupting BLE radio from low power BLE: Sleep Mode to BLE: TX Beacon Mode. In case of opportunistic BLE beacons operation, $\mathrm{AB}$ and $\mathrm{AS}$ are not synchronized; so these activities must overlap for beacon discovery to initiate. Figure 4 shows a beacon timing required to establish a reliable opportunistic beacon network, where: $T_{B C}^{+}$- advertising interval, $T_{s c}^{+}$- scanning interval, and $T_{s W}^{+}$- scanning window, where $T_{s W}^{+} \geq T_{B C}^{+}$ for the AS node to pickup the AB BLE beacons in the area. Table I summarizes the BLE beacon communication timings involved.

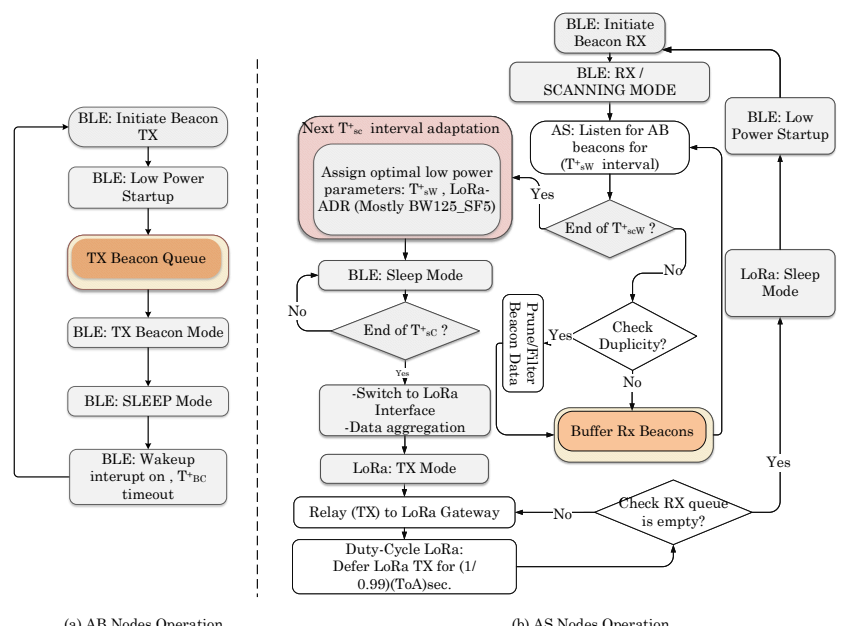

Fig. 3: $A B$ and $A S$ node operation flowchart.

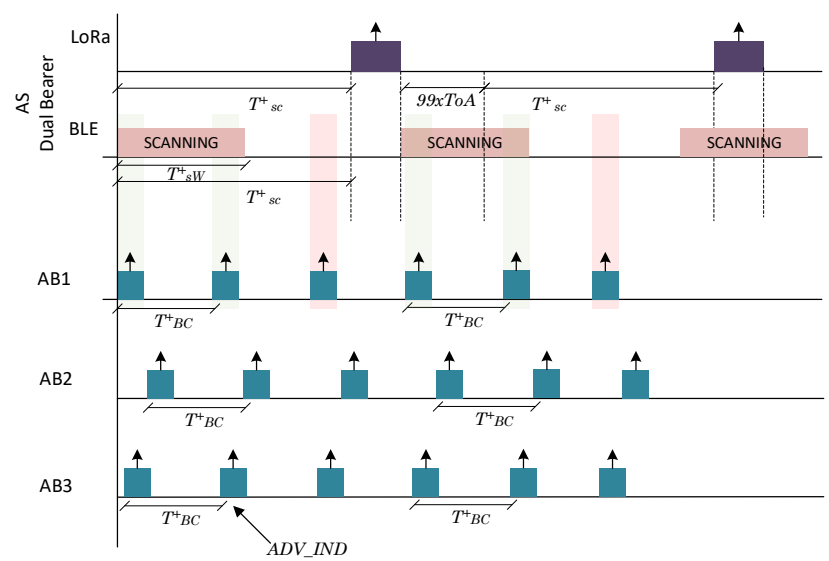

Fig. 4: Data transmission timing on AS node with dual interface (BLE and LoRa). The AS node concatenates AB beacon packets and relays it through LoRaWAN every $T_{s c}^{+}$while obeying the $1 \%$ dutycycle restriction. BLE AB-to-AS Timing: $T_{B C}^{+}$-advertiser interval, $T_{s c}^{+}$ -scanner interval, and $T_{s W}^{+}$-scanner window, where $T_{s W}^{+} \geq T_{B C}^{+}$.

TABLE I: BLE Beacon Timing

\begin{tabular}{lll}
\hline Notation & Meaning & Recommendation \\
\hline$T_{B C}^{+}$ & Adv. Interval & $\begin{array}{l}\text { Int. multiple } 0.625 \mathrm{~ms} \\
\text { in }[20 \sim 10240] \mathrm{ms}\end{array}$ \\
\hline$\beta_{(B d)}$ & Uniform random delay & {$\left[0 \sim \beta_{\left.B d_{\max }\right]}\right.$} \\
\hline$\beta_{B d_{\max }}$ & Upper delay bound & $\beta_{B d_{\max } \leq 10 \mathrm{~ms}}$ \\
\hline$T_{s c}^{+}$ & Scan Interval & Integer multiple of $0.625 \mathrm{~ms}$ in \\
\hline \multirow{2}{*}{$T_{s w}^{+}$} & & {$[2.5 \sim 10240] \mathrm{ms}$} \\
\hline & Scan Window & Integer multiple of $0.625 \mathrm{~ms} \mathrm{in}$ \\
& & {$[2.5 \sim 10240] \mathrm{ms}$,} \\
& & $T_{s W}^{+} \leq T_{s c}^{+}$ \\
\hline
\end{tabular}

Similarly, as illustrated in Figure 3b, AS node also commences its periodic scanning operation by changing its BLE radio from BLE: Low Power Mode to BLE: RX Scanning Mode. An AS device starts the beacon scanning with predefined 
default $T_{s w}^{+} \geq T_{s, \text { min }}^{+}$values, to allow the asynchronous $\mathrm{AB}$ beacon data to overlap with the listening window of $\mathrm{AS}$ node. To cope with the variable number of incoming $A B$ beacons, AS node adapts the scanning time interval based on the number of beacons received from $\mathrm{AB}$ nodes. AS node listens and keeps track of the number of beacons it has received during its current and previous scan period $\left(T_{s w}^{+}\right)$with (prevBeaconNum and curBeaconNum) variables. Each AS node compares its (prevBeaconNum) with curBeaconNum top decide the length of the next scanning interval. This approach will contribute to a reduced energy consumption of AS node by adaptively controlling the scanning interval $\left(T_{s w}^{+}\right)$. Algorithm 1 summarizes this procedure.

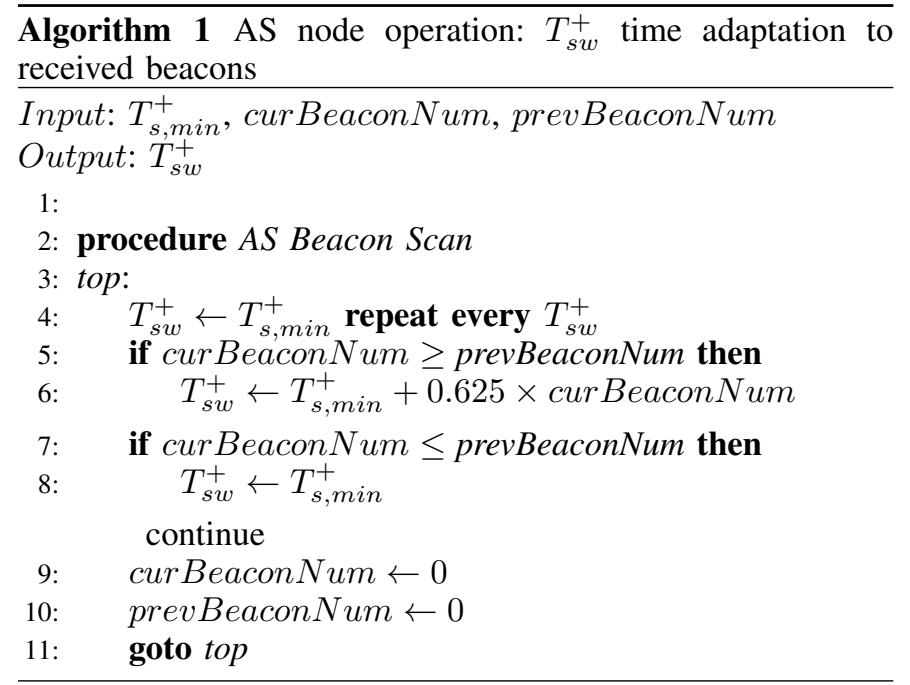

This $T_{s, \text { min }}^{+}$is equal to the recommended minimum broadcast interval $\left(T_{B C}^{+}=100\right)$ in BLE protocol specification for beacon functionality. When (curBeaconNum $\geq$ prevBeaconNum), then AS updates its next service to start with longer scanning window of $\left(T_{s W}^{+}=T_{s, \min }^{+}+0.625 \times\right.$ curBeaconNum), where, $\left[2.5 \leq T_{s W}^{+} \leq 10240\right] \mathrm{ms}$ and $T_{s W}^{+} \leq T_{s c}^{+}$as per BLE specification [15]. The longer the $T_{s W}^{+}$ interval, the more beacon it can listen in one period. In case of (curBeaconNum $\leq$ prevBeaconNum), $T_{s w}^{+}$is by default $T_{s w}^{+}=T_{s, \text { min }}^{+}$to start over the periodicity. In this way, AS adapts its scanning time according to the dynamic number of beacons it receives.

Figure 4 shows the operation of AS nodes. At the end of every scanning window $\left(T_{s w}^{+}\right)$, AS periodically relays processed $\mathrm{AB}$ beacon data to LoRaWAN network by utilizing its LoRa radio interface. While leveraging LoRa the AS node is restricted by the $1 \%$ LoRa transmission duty-cycle regulation [3]. This restriction and node mobility could contribute to high network latency. For example, for LoRa payload of $P L=51$ bytes using LoRaWAN star topology ( $\mathrm{SF}=7, \mathrm{CR}$ $=1, \mathrm{DR}=5 \mathrm{kbps}$ ), the time on air will be $T o A=71.936 \mathrm{~ms}$ and AS node has to deffer sending for $T_{\text {off }} \approx 7.1936 \mathrm{~s}$, which is practically long for real-time (fine-grained) monitoring of mobile network environment with frequently changing RSSI values [3].

To alleviate this issue, the proposed approach utilizes data merging and pruning at AS node to reduce latency. Therefore, AS encodes several beacons to LoRa packet to be relayed to
LG (see Figure 3b). At the end of every scanning interval, AS node turns off LoRa interface and again switches back to BLE interface to continue receiving the BLE beacons while complying to $1 \%$ duty-cycle regulation.

\section{OPTIMAL BEACON TRANSMISSION INTERVALS REQUIREMENT OPTIMIZATION}

The AB node's beacon advertising interval $\left(T_{B C}^{+}\right)$value for $\mathrm{AB}$ nodes have an impact on application requirements of a given opportunistic beacon network protocol. Achieving high average delivery ratio $\left(D_{e}\right)$, low average latency $(\ell)$, and high average network life-time $\left(N_{l}\right)$ are the main requirements often considered [3]. Hence, in this section, we formulate these requirements as an optimization challenge and discuss its practicality.

Let $S_{r} \subset N$ denote set of $\mathrm{AB}$ beacon generating nodes in a network with $N$ number of $\mathrm{AB}$ nodes. $L$ denotes the set of wireless (AB to $\mathrm{AS}$ ) links. The link $L_{i} \subset L$ originating from node $i \in S_{r}$ is the link that connects AB node $i$ to AS node. Hence, the beacon network requirement optimization could be expressed as:

$$
\begin{array}{ll}
\underset{\ell \in L_{i}}{\operatorname{Maximize}} & D_{e}=\frac{1}{\left|S_{r}\right|} \sum_{i \in S_{r}} D_{e L_{i}}=\frac{1}{\left|S_{r}\right|} \sum_{i \in S_{r}} P_{i} \\
\text { Subject to } & D_{e} \geq D_{e \min }, i=1, \ldots, N . \\
\ell & =\frac{1}{\left|S_{r}\right|} \sum_{i \in S_{r}} \ell_{L_{i}+L_{L o R a}} \leq \ell_{\max } \\
& N_{l}=\frac{1}{\left|S_{r}\right|} \sum_{i \in S_{r}} N_{l_{i}} \geq N_{l_{\text {min }}}
\end{array}
$$

Where per AB-AS link delivery ratio $D_{e L_{i}}$ of link $L_{i}$ is the expected beacons successfully delivered from AB node $i \in S_{r}$ to AS node along $L_{i}$ link. Then average delivery ratio $\left(D_{e}\right)$ is defined as the average of all links $L_{i}$ (See Eq. 1). $D_{e}$ is simplified further as the probability $P_{i}$ that $\mathrm{AB}$ node $i$ will successfully deliver to AS.

Similarly, we define the per-hop latency $\ell_{L_{i}}$ of link $L_{i}$ as the time for $\mathrm{AB}$ node $i$ to deliver a beacon to AS. In addition a significant latency overhead is introduced due to the $1 \%$ LoRaWAN duty-cycle regulation $\left(L_{L o R a}\right)$, when at the end of every $T_{s C}^{+}$, AS node utilizes LoRa interface to relay data to the LG node. $L_{L o R a}$ is directly related to the time-on-air (ToA) of the defined LoRa packet payload $(P L)$. Thus, as shown in Eq. 1, the total average end-to-end network latency $(\ell)$ is expressed as a total average of $\ell_{L_{i}}$ and $L_{L o R a}=T_{s C}^{+}+99 \times$ ToA. Furthermore, the Network life-time $\left(N_{l}\right)$ is also defined as the average time before individual nodes $\left(N_{l_{i}}\right)$ stops operating (see Eq. 1).

Therefore, to satisfy the given beacon network requirements, an upper bound could be introduced on the acceptable level of maximum latency, $\ell \leq \ell_{\max }$, for a timely data delivery. Likewise, an applicable minimum average delivery ratio, $D_{e} \geq D_{e m i n}$, and minimum per node-life-time $N_{l_{i}} \geq N_{l m i n}$ could be defined.

Since the performance of $\mathrm{AB}$ beacon advertising primarily depends on $\left(T_{B C}^{+}\right)$, thus, $\mathrm{AB}$ nodes should be configured with 
an optimal $T_{B C}^{+}$beacon interval to satisfy the network requirements. To solve Eq. 1, we define a simple mathematical model to describe the AS device discovery latency, and characterize the collision probability and/or reliability according to the ideal implementation of BLE specification [15]. Note that the beacon collision probability depends on three main factors: (i) collisions between $\mathrm{AB}$ beacon packets, (ii) $\mathrm{AB}$ beacon timing parameter configurations and (iii) channel and interference conditions.

Thus, we model the probability of how likely it is that an $\mathrm{AB}$ device send an $\mathrm{AB}$ beacon packet $\mathrm{AS}$ without a collision. Given that $T_{B C}^{+}$is beacon interval and $\Gamma$ is the duration of $\mathrm{AB}$ beacon data. The number of BLE devices involved in the analysis is $N+1$, i.e. a device is configured as a scanner (AS), whereas the other $N$ devices are configured as advertisers (ABs). Hence, $\mathrm{AB}$ beacon data will overlap and create collision, if it starts anywhere in $\Gamma$ duration, inclusive of before $\mathrm{AB}$ starts up until when it finishes advertising $[0, \Gamma]$, then it will be an overlap window of $2 \Gamma$ or $[0,2 \Gamma]$ length, where there is a chance of collision.

$$
P_{i}(\forall(N-1))=\left(1-2 \Gamma / 3 T_{B C}^{+}\right)^{(N-1)}
$$

For $N$ number of $\mathrm{AB}$ nodes in the network and assuming the best case scenario, (i.e. the transmitted $\mathrm{AB}$ beacons are all successfully received by the AS node), the probability that $i^{t h}$ node's beacon misses (no collision) all other $(\mathrm{N}-1)$ AB's beacons in the same channel is $P_{i}(\forall A B)=P(n o-$ collision $)^{(N-1)}$, as expressed in Eq. 2 .

Thus, the average network reliability $(R)$ for the beacon network, would be $D_{e}=\frac{1}{N}\left(\sum_{i \in N-1} P_{i}(\forall A B)\right)$. This generalization holds true even when multiple AS nodes exit in the same radio coverage area, since BLE beacon is based on broadcast communication mode where multiple $\mathrm{AS}$ and $\mathrm{AB}$ nodes share the same channel.

Similarly, the expected per-link $i^{\text {th }}$ beacon discovery latency at AS is given by:

$$
\ell_{L_{i}}=\left[T_{B C}^{+}+\beta_{B d_{\max }}+P_{i}(\forall(N-1)) \times(\Gamma)\right] \times 10^{3}[m s]
$$

Thus, the average network latency $(\ell)$ for the beacon network, would be $\ell=\frac{1}{N}\left(\sum_{i \in N-1} \ell_{L_{i}+L_{L o R a}}\right)$.

In addition, given a battery capacity $Q_{p}[m A h], E$ is the average energy consumption, and supply voltage $(v)$, the individual $i^{t h} \mathrm{AB}$ node's life-time $\left(N_{l_{i}}\right)$ is expressed as:

$$
N_{l_{i}}=\left(\frac{Q_{p} \times V}{E_{i} \times T_{B C}^{+}}\right)
$$

Likewise, the average network node-life would be $N_{l}=$ $\left(\frac{1}{N}\right)\left(\sum_{i=1}^{N} N_{l_{i}}\right)$, where $E=\Gamma_{i} \times P_{t_{i}}, P_{t_{i}}$ is the transmission power, $\mathrm{N}$ is the number of end-nodes.

Hence, finding the optimal $T_{B C}^{+}$, is straight forward given the required expected delivery ratio $\left(D_{e}\right)$, latency $(\ell)$, and network node-life $\left(N_{l}\right)$, by averaging Eq. 2, Eq. 3, and Eq. 4 for $N$ AB nodes, respectively. This approach is demonstrated in the evaluation section.

\section{EVALUATION}

In order to evaluate the proposed protocol for large scale scenario, a realistic simulation environment is setup. To investigate its suitability for animal monitoring applications, we compare our approach with conventional opportunistic protocols, such as Epidemic, and ProPHET in-terms of network life-time (energy) and packet delivery ratio as described in our previous work [5].

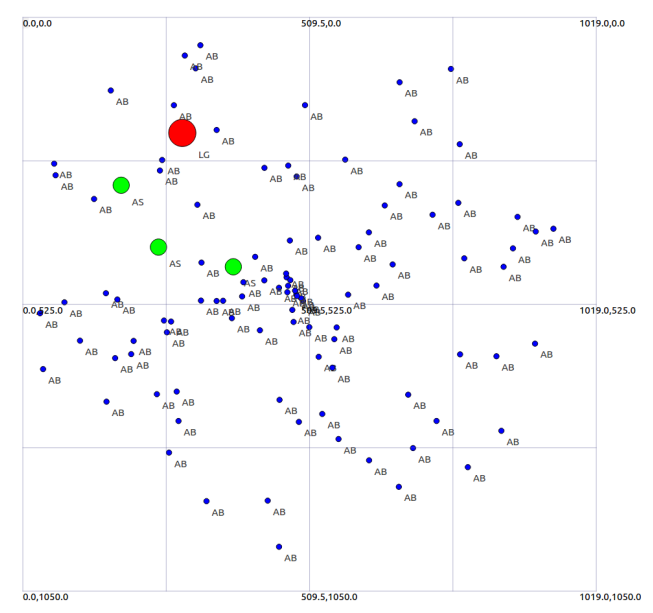

Fig. 5: Simulation Setup With Dual Interface NS3 Simulation Environment. Color labels; $B L U E=A B, G R E E N=A S, R E D=L G$ nodes

\section{A. Simulation Set-up}

We evaluate the performance of the protocol in the NS3 simulation environment [16]. Figure 5 shows the simulation setup for NS3 deployments with $70 \mathrm{AB}$ and 3 AS nodes moving in a defined trajectory in a grid area of $1000 \mathrm{mx} 1000 \mathrm{~m}$, and one LoRaWAN gateway is used to receive data from the AS node. However, we also ran the simulation for a range of parameters as summarized in Table II.

$\mathrm{AB}$ nodes generate beacons with 31 bytes (i.e. max BLE payload). We configured $N$ number of $\mathrm{AB}$ and $N_{A S}$ of AS devices in NS3 simulator. To investigate the effect of $T_{B C}^{+}, T_{s c}^{+}$, and $T_{s W}^{+}$on the network performance metrics, we run several simulations, where $\mathrm{AB}$ nodes transmit beacon at varying $T_{B C}^{+}$ and AS nodes perform scanning with a particular $T_{s c}^{+}$, and $T_{s W}^{+}$ settings.

For each $T_{B C}^{+}$, we use the values in steps of $100 \mathrm{~ms}$ in [100 600] ms. To make sure that AS and AB timing overlap, we follow the BLE timing guide line, i.e. for $T_{s c}^{+}$values of setting $700 \mathrm{~ms}, 800 \mathrm{~ms}, 1000 \mathrm{~ms}$ and $T_{s W}^{+}$values of setting $T_{s W}^{+} \gg T_{B C}^{+}+10,600 \mathrm{~ms}, 700 \mathrm{~ms}$. While, this setting is not optimal for power, it is useful to test the beacon packet collision and delivery.

We recorded the packet generation time at $\mathrm{AB}$ nodes, as well as the time when they are received at the LoRaWAN gateway. The simulation measurement is performed for a total of $15 \mathrm{hr}$ simulation time. In our simulation animals are assumed to be mobile, hence, we introduce a mobility model $\left(M_{1}\right)$ for group (herd) of animal movement from the ZebarNet 
TABLE II: Simulation Input Parameters

\begin{tabular}{c|c}
\hline Mobility Model $\left(M_{1}\right)$ & $M_{o}^{\perp}:$ ZebarNet \\
\hline Freq. & $1 \%(\mathrm{LoRa})$ \\
\hline Duty-cycle & $4 / 5(\mathrm{LoRa})$ \\
\hline Coding Rate & $125 \mathrm{KHz}(\mathrm{LoRa})$ \\
\hline Bandwidth & $7-12(\mathrm{LoRa})$ \\
\hline Spreading Factor (SF) & $125 \mathrm{KHz}(\mathrm{LoRa})$ \\
\hline Bandwidth & BLE $(1 \mathrm{Mbps})$ \\
\hline Data Rate (DR) & $4 \mathrm{Bmm}(\mathrm{BLE}, \mathrm{LoRa})$ \\
\hline$P_{t}$ & $N^{0}: 15, N^{1}: 50$, \\
\hline$A B$ Node Density $(N)$ & $N^{2}: 100, N^{3}: 150, N^{4}: 200, N^{5}: 250$, \\
& $N^{6}: 300, N^{7}: 350, N^{8}: 400$ \\
\hline$A S$ Node Density $\left(N_{A S}\right)$ & $N_{A S}^{0}: 1, N_{A S}^{1}: 3$ \\
\hline Simulation Area $\left(S_{A}\right)$ & $500 \mathrm{mx} 500 \mathrm{~m}$ \\
\hline Packet $(\mathrm{PL})$ & 31 bytes $(\mathrm{Max}$ for ADV) \\
\hline$T_{B C}^{+}$ & {$[100 \sim 600] \mathrm{ms}$ in $100 \mathrm{~ms}$ steps } \\
\hline$T_{s c}^{+}$ & $700 \mathrm{~ms}, 800 \mathrm{~ms}, 1000 \mathrm{~ms}$ \\
\hline$T_{s W}^{+}$ & $600 \mathrm{~ms}, 700 \mathrm{~ms}$ \\
\hline Simulation duration $(\mathrm{hr})$ & 15 \\
\hline
\end{tabular}

project, generated from real GPS data [17]. For Epidemic and ProPHET protocols the replication data is set to TTL $=10 \mathrm{~s}$. Other parameters are set same as in Table II. Our protocol uses a single copy replication with data aggregation and pruning at the AS node. While the other protocols use multicopy replication without data processing at AS node, which will have an impact on the network performance e.g. energy, latency and reliability measures as it will be demonstrated in the following section.

\section{B. Performance Metrics}

Three metrics are used to evaluate the proposed network as outlined in Section IV:

- Average Delivery Ratio $\left(D_{e}\right)$ is a measure of the ratio of number of beacon packets successfully received by a loRaWAN gateway ( $\mathrm{LG}$ ) to number of $\mathrm{AB}$ beacons sent. In NS3, we count the number of LoRa packets received at the LG node and the total number of $\mathrm{AB}$ beacons sent.

- End-to-End Latency $(\ell)$, since beacon network is onehop communication a unidirectional average latency of a beacon defines the ratio of the time when the beacon is transmitted to the time when it is received at LG. Hence, the $\ell$ will be highly influenced by the Time-On-Air (ToA) of a LoRaWAN packets and 1\% duty-cycle limitation imposed. NS3 records the time when a packet is received at the LG node and the time when it is sent to determine the $\ell$.

- Network life-time $\left(N_{l}\right)$, is a function of average energy consumption of all nodes, as defined in Equation 4.

\section{Result and Discussion}

In this section, we evaluate the network in comparison to existing opportunistic networks in NS3.

Figure 6 shows that our approach performs better than Epidemic and ProPHET network in terms of average data delivery ratio $\left(D_{e}\right)$. The main reason for this is that Epidemic and ProPHET have a multiple copy data delivery approach compared to our single copy approach. This will create a high collision at the receiver nodes. Hence, they have lower
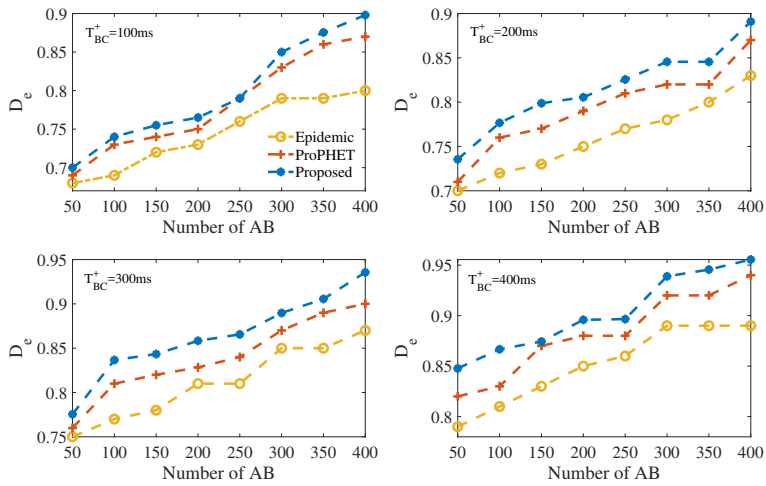

Fig. 6: Comparison of average delivery ratio $\left(D_{e}\right)$ for proposed, Epidemic, and ProPHET opportunistic protocols in ZebarNet mobility scenario: with $T_{s c}^{+}=700 \mathrm{~ms}$, and $T_{s W}^{+}=600 \mathrm{~ms}$ for variable number of $A B$ nodes.
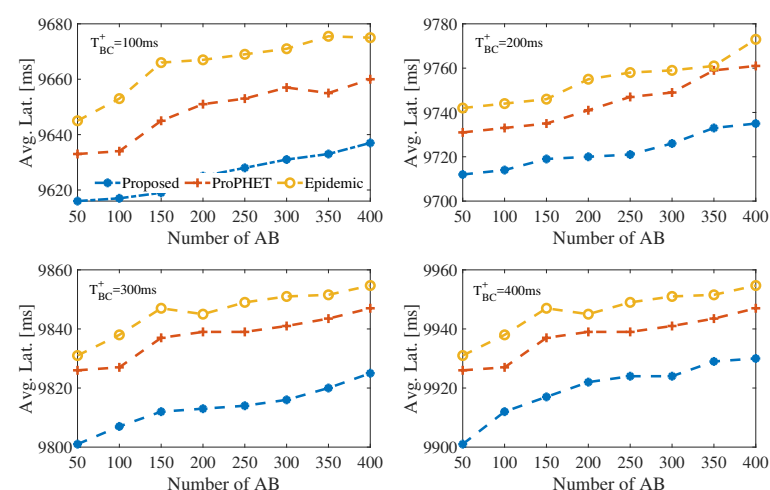

Fig. 7: Comparison of average latency $(\ell)$ for proposed, Epidemic, and ProPHET opportunistic protocol in ZebarNet mobility scenario: $T_{s c}^{+}=700 \mathrm{~ms}$, and $T_{s W}^{+}=600 \mathrm{~ms}$ for variable number of $A B$ nodes.

probability of data delivery than our proposed protocol. Moreover, Epidemic and ProPHET are often demand higher network resources such as buffer and battery, which are very scarce in the wildlife monitoring applications, this result in higher latency and high energy consumption (Fig. 7). As shown in Figure 8, the network life-time is very short for Epidemic, due to the same reason that it increases the communication overhead than the our simplified protocol [5].

Choosing the right $\mathrm{AB}$ beacon timing parameters, $\left(T_{B C}^{+}\right.$, $T_{s c}^{+}$, and $T_{s W}^{+}$), should be based on application requirements. Both fast or slow beacon modes have advantage and disadvantages. For instance, longer $T_{B C}^{+}$duration is slower beaconing and have a lower power consumption, consequently, low probability of short discovery time by AS nodes. While shorter $T_{B C}^{+}$duration, for fast beaconing results in a higher power consumption, but with high probability of short discovery time by AS nodes. In a time-constrained applications as in WMS, when the AS needs to receive data as close as real-time, then $T_{s W}^{+} \gg T_{B C}^{+}+\beta$, to guarantee discovery. Moreover, to prevent advertising events from multiple beacons from colliding, a small random time $(\beta=[0-10] \mathrm{ms})$ is added between advertising events. Adhering to this beacon timing guide line 
will reduce beacon collision and increase the delivery ration, as summarized in table I.

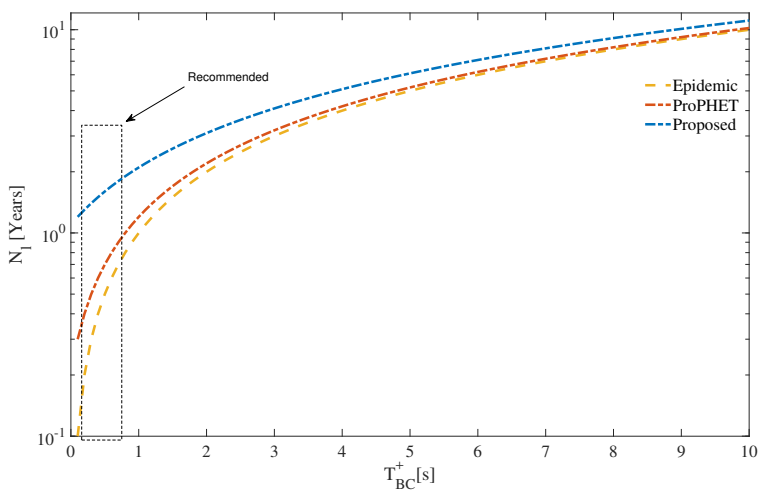

Fig. 8: Comparison of network life-time $\left(N_{l}\right)$ for proposed, Epidemic, and ProPHET opportunistic networks with ZebarNet mobility scenario for $V=1.225 \mathrm{v}, Q_{p}=1150 \mathrm{mAh}$.

Figure 8 shows the average network life time assuming all $\mathrm{AB}$ nodes in the beacon network are configured with same $T_{B C}^{+}$settings. The network life-time is independt of the number of $\mathrm{AB}$ nodes in the network, however, it highly depends on the value of advertising interval set $\left(T_{B C}^{+}\right)$. One of important issue to realize is the trade-off between power consumption and latency. Generally, the less frequent advertisements, the longer the beacon network runs (Figure 8). For example, if the total number of $\mathrm{AB}$ nodes in the network is $N=200$, therefore, in order to insure a network life-time $N_{l}=4$ years, average delivery ratio $D_{e}=80 \%$ and average discovery latency of $\ell \approx 9000 \mathrm{~ms}$, thus, the optimal $T_{B C}^{+}$would be in the range of $T_{B C}^{+}=\approx[200,300] \mathrm{ms}$ (as per Figure 6,7 and 8). Hence, the optimal value of $T_{B C}^{+}$should be chosen depending on $N$ and the required beacon network performance measures, to optimally reduce collision in beacon network.

\section{CONCLUSION}

In this paper, we presented a simple asynchronous dual interface network architecture for animal monitoring. The key advantage of this architecture is that nodes achieve wider control on the trade-off between total energy consumption and latency. The evaluation results show that the proposed architecture outperforms the traditional opportunistic networks. On average, our protocol improved the data delivery radio and latency incured by up-to $60 \%$ and $75 \%$ respectively. In addition, the architecture improved the network life time by up-to $50 \%$ especially for the faster packet traffic rates in the network. Therefore, our protocol is more optimal to deploy than utilizing only conventional opportunistic network. Moreover, in the future, we plan to implement this architecture in the real world sensor devices, by building a prototype with dual radios.

\section{ACKNOWLEDGMENT}

This research was supported by Smart Parks Project, funded by the Netherlands Organization for Scientific Research (NWO).

\section{REFERENCES}

[1] Paul O'Donoghue and Christian Rutz. Real-time antipoaching tags could help prevent imminent species extinctions. Journal of Applied Ecology, 53(1):5-10, 2016.

[2] William E Cooper Jr. Escaping from predators: an integrative view of escape decisions. Cambridge University Press, 2015.

[3] E. D. Ayele, K. Das, N. Meratnia, and P. J. M. Havinga. Leveraging ble and lora in iot network for wildlife monitoring system. In 2018 WF-IoT, pages 342-348, Feb 2018.

[4] Shih-Lin Wu and Yu-Chee Tseng. Wireless ad hoc networking: personal-area, local-area, and the sensoryarea networks. CRC Press, 2007.

[5] Eyuel D Ayele, Nirvana Meratnia, and Paul JM Havinga. Towards a new opportunistic iot network architecture for wildlife monitoring system. In NTMS, 2018 9th, pages 1-5. IEEE, 2018.

[6] S. Pathak, N. Gondaliya, and N. Raja. A survey on prophet based routing protocol in dtn. In ICEI, pages 110-115, Feb 2017.

[7] P. Juang, H. Oki, Y. Wang, M. Martonosi, Li S. Peh, and D. Rubenstein. Energy-efficient computing for wildlife tracking: Design tradeoffs and early experiences with zebranet. In ACM Sigplan Notices, volume 37, pages 96-107. ACM, 2002.

[8] O. Landsiedel, J gila B., K Wehrle, J. Thiele, and H. Mallot. Rat watch. ACM REALWSN, 2006.

[9] and. Wetlands study in china: creating a database using gps and gis technology. IEEE Instrumentation Measurement Magazine, 8(4):40-43, Oct 2005.

[10] Aruna Balasubramanian, Brian Levine, and Arun Venkataramani. Dtn routing as a resource allocation problem. In Proceedings of the 2007 Conference on Applications, Technologies, Architectures, and Protocols for Computer Communications, SIGCOMM '07, pages 373-384, New York, NY, USA, 2007. ACM.

[11] A. Al-Hinai, H. Zhang, Y. Chen, and Y. Li. Tb-snw. The Journal of Supercomputing, 69(2):593-609, Aug 2014.

[12] S. Raza, P. Misra, Z. He, and T. Voigt. Building the internet of things with bluetooth smart. Elsevier, Ad Hoc Networks, 57:19-31, 2017.

[13] N. Sornin, M. Luis, T. Eirich, T. Kramp, and O. Hersent. Lorawan specifications. LoRa Alliance, 2015.

[14] M. Centenaro, L. Vangelista, A. Zanella, and M. Zorzi. Long-range communications in unlicensed bands: the rising stars in the iot and smart city scenarios. IEEE Wireless Communications, 23(5):60-67, October 2016.

[15] Ble core specifications, September 2018. [online] https://www.bluetooth.com/specifications/ bluetooth-core-specification.

[16] Ns3 network simulator, Mar. 2019. [online] https://www. nsnam.org/.

[17] Philo Juang, Hidekazu Oki, Yong Wang, Margaret Martonosi, Li Shiuan Peh, and Daniel Rubenstein. Energy-efficient computing for wildlife tracking. ACM SIGOPS Operating Systems Review, 36(5):96, 2002. 